\title{
Investigation of Schema Therapy Performance in Borderline Personality Disorder Patients
}

\section{Elham Mohammadipour ${ }^{1}$}

*This Article has remove from this issue. Because, this article under the copyright issue and plagiarism.

** This article has been retracted at the request of the Editor-in-Chief.

Please note: The International Journal of Indian Psychology (IIIP) provides platform for researchers to publish and discuss their original research and review work. IJIP cannot be held responsible for views, opinions and written statements of researchers published in the journal. IJIP strongly condemn and discourage practice of plagiarism. 